\title{
Karpaltunnelsyndrom - ein Fall aus der Praxis
}

\author{
Benjamin Neul, Carina Jensen
}

\begin{abstract}
Frau S. litt nach längerem Schreiben unter Schmerzen und Schwäche der Hand. Durch die andauernde Belastung hatte sich ein Karpaltunnelsyndrom gebildet. Eine veränderte Stifthaltung und die Stärkung des M. flexor pollicis longus schafften Abhilfe.
\end{abstract}

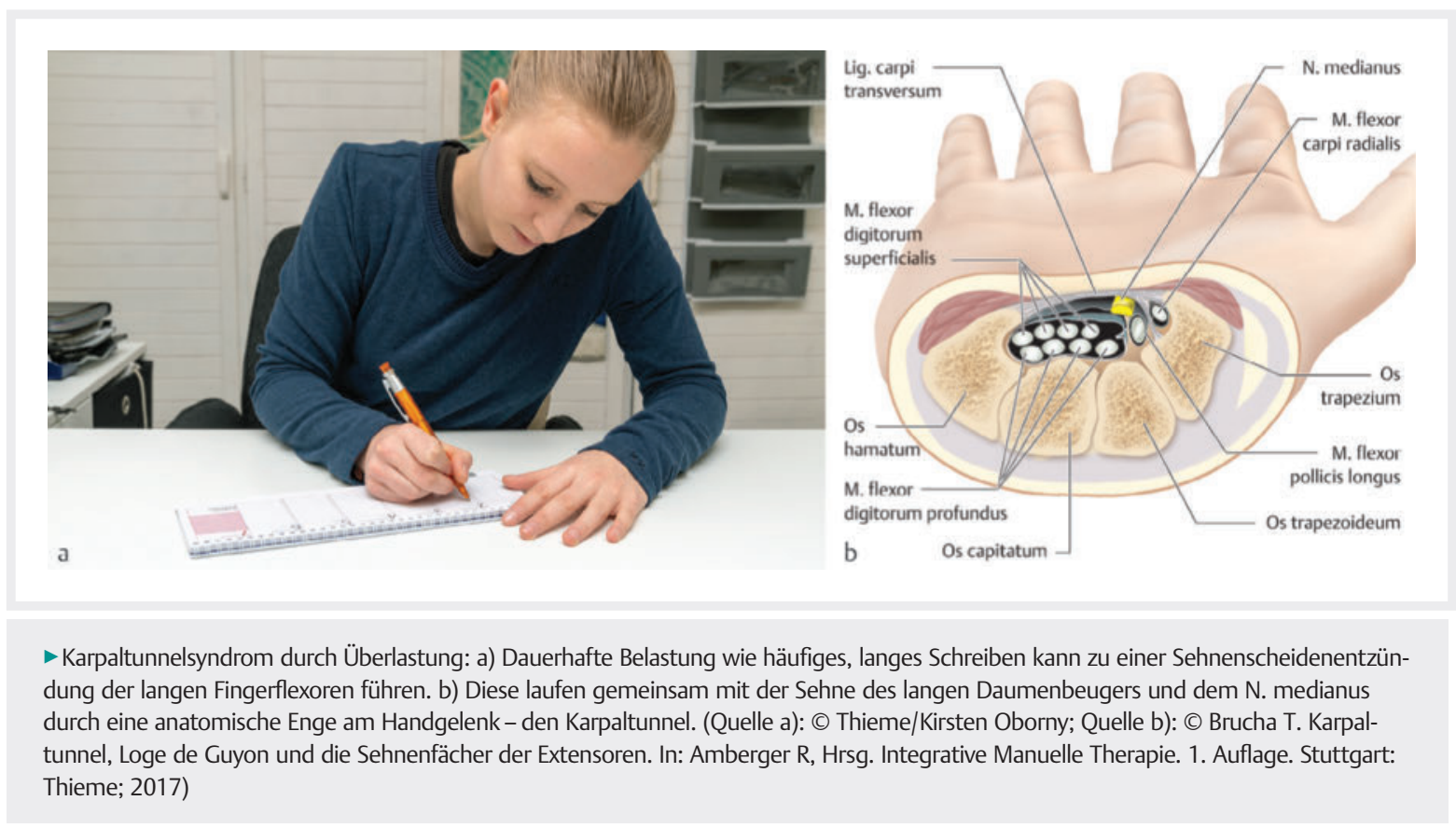

Der Karpaltunnel ist ein osteofibröser Kanal, der durch die Handwurzelknochen und das Retinaculum flexorum begrenzt bzw. gebildet wird. Er dient als Platzhalter für die Beugesehnen der Finger und des Daumens (Mm. flexor digitorum profundus und superficialis sowie M. flexor pollicis longus) und den N. medianus. Dieser innerviert die palmare Seite der Finger 1-3 sensibel. Motorisch versorgt er vor allem die Flexoren des Unterarms (mit Ausnahme des M. flexor digitorum profundus $4+5$ ) und Teile der Thenarmuskulatur: Mm. abductor pollicis brevis, flexor pollicis brevis (Caput superficiale), opponens pollicis sowie die Mm. lumbricales $1+2$.

Wird es dem N. medianus innerhalb des Kanals zu eng, entsteht ein Karpaltunnelsyndrom (KTS). Erkennbar wird dies vorwiegend anhand von Einschlafgefühlen und schmerzhaften Missempfindungen der Finger 1-3, die besonders nachts auftreten und durch Ausschütteln der Hand reduzierbar sind. Im fortgeschrittenen Stadium kann es zudem zu sensomotorischen Ausfällen sowie zu einer Atrophie des Thenars kommen.

Das KTS gilt als das häufigste Nervenkompressionssyndrom der oberen Extremität, an dem Frauen etwa 3-4 Mal häufiger erkranken als Männer. Mit einer Prävalenz von $80 \%$ sind Patienten meistens beidseits (zeitversetzt) betroffen [1].

\section{Ursachen}

Das Engpasssyndrom des N. medianus kann aufgrund diverser anatomischer Veränderungen oder durch eine Volumenzunahme im Tunnel entstehen. Die verschiedenen Ursachen reichen von tumorähnlichen Raumforderungen (z. B. Ganglion im Bereich oder am Rand des Karpaltunnels) bis hin zu einer Luxation eines Handwurzelknochens. Hierbei ist häufig das Os lunatum betroffen, das aufgrund eines Traumas seine Position verändern und 
den N. medianus irritieren kann. Aber auch Veränderungen des Hormonhaushaltes (Schwangerschaft, Menopause) können durch Wassereinlagerungen im Bereich der Hand ein KTS hervorrufen. Ist der Zustand nur vorübergehend (Schwangerschaft), lohnt es sich, diese Zeit konservativ mittels Lymphdrainage zu überbrücken. Durch den Abtransport der überschüssigen Lympheinlagerung kann das KTS oft gelindert oder gar behoben werden.

In den meisten Fällen jedoch entsteht die Enge aufgrund einer Tendovaginitis des 3. palmaren Sehnenfachs, das die Sehnen der Mm. flexor digitorum superficialis und profundus (FDS/FDP) führt. Die entzündlichen Veränderungen sind dabei auf degenerative, rheumatische oder metabolische Erkrankungen (rheumatoide Arthritis, Diabetes mellitus) zurückzuführen. Auch eine Überlastung und der damit verbundene Hypertonus der Unterarmflexoren kann zur Volumenzunahme und Reizung der Sehnen innerhalb des Tunnels führen.

Proximal des Karpaltunnels kann der N. medianus beispielsweise durch einen Hypertonus oder eine Hypertrophie des M. biceps brachii (der N. medianus zieht unter dem Lacertus fibrosus der Bizepssehne hindurch) oder des M. pronator teres (sog. Pronator-teres-Syndrom) komprimiert werden.

Neben den peripheren Ursachen sollten Therapeuten auch das Thoracic-Outlet-Syndrom, Radikulopathien der Halswirbelsäule (HWS) und Polyneuropathien als Differenzialdiagnosen in Betracht ziehen. Innerhalb der klinischen Untersuchung bedarf es daher einer Kombination aus verschiedenen Testverfahren, um ein Karpaltunnelsyndrom und dessen beitragende Faktoren zu klassifizieren.

\section{Therapeutische Diagnostik}

Um die Diagnose zu sichern und um spezifische Ursachen für das Auftreten der Symptome zu erkennen, ist häufig „Detektivarbeit“ gefragt, an der Patienten beteiligt werden sollten. Durch eine gezielte Anamnese im Hinblick auf
Ergonomie am Arbeitsplatz oder Überlastung im Haushalt bzw. im Handwerk gewinnen Therapeuten behandlungsrelevante Schlüsselinformationen. Im Zuge dessen sind zur Evaluation von Alltagseinschränkungen und zur OutcomeMessung der Boston Carpal Tunnel Questionnaire (BCTQ) sowie der Disabilities of Arm, Shoulder and Hand Questionnaire (DASH) empfehlenswert [2].

Im weiteren Verlauf erfolgt eine Funktionsuntersuchung zur Überprüfung der Beweglichkeit, Kraft und Sensibilität. Neben der aktiven und passiven Bewegungsprüfung der HWS (Symptome könnten im Falle einer Radikulopathie reproduziert werden) und des Handgelenkes (Überprüfung der Sehnenscheiden) sollten Therapeuten eine Instabilitätstestung der Handwurzelknochen durchführen, um eine Subluxation des Os lunatum im Karpaltunnel auszuschließen. Isometrische Krafttests für die Ellenbogenflexion und Supination (Provokation bei Hypertonus des M. biceps brachii) sowie Pronation (Provokation des $M$. pronator) sind Bestandteile der therapeutischen Differenzialdiagnostik.

Um die Sensibilität der Hand zu prüfen, haben sich vor allem 2 Tests bewährt: der Semmes-Weinstein Test, der die Wahrnehmungsschwelle über ein kalibriertes Monofilament misst, und die Zwei-Punkt-Diskrimination, bei der evaluiert wird, inwieweit Patienten 2 taktile Reize noch getrennt voneinander wahrnehmen können.

Weiterhin sind Provokationstests (Hoffmann-Tinel-Zeichen, Phalen-Test und Durkan-Test, > Abb. 1) zur Diagnostik empfehlenswert. Während Therapeuten beim Tinel-Zeichen den Karpaltunnel in leicht vorpositionierter Dorsalextension der Hand mehrfach mit ihren Fingern beklopfen, flektieren Patienten beim Phalen-Test einoder beidseitig aktiv ihr Handgelenk für mindestens eine Minute. Beim Durkan-Test komprimiert der Untersucher den Karpaltunnel für etwa 30 Sekunden mit beiden Daumen. Hierbei befindet sich der Ellenbogen des Patienten in Extension, der Unterarm in Supination und das Handgelenk in ca. $60^{\circ}$ Palmarflexion. Die Tests gelten als positiv, wenn sie die für den Patienten typischen Parästhesien reproduzieren. Die jeweiligen Ergebnisse
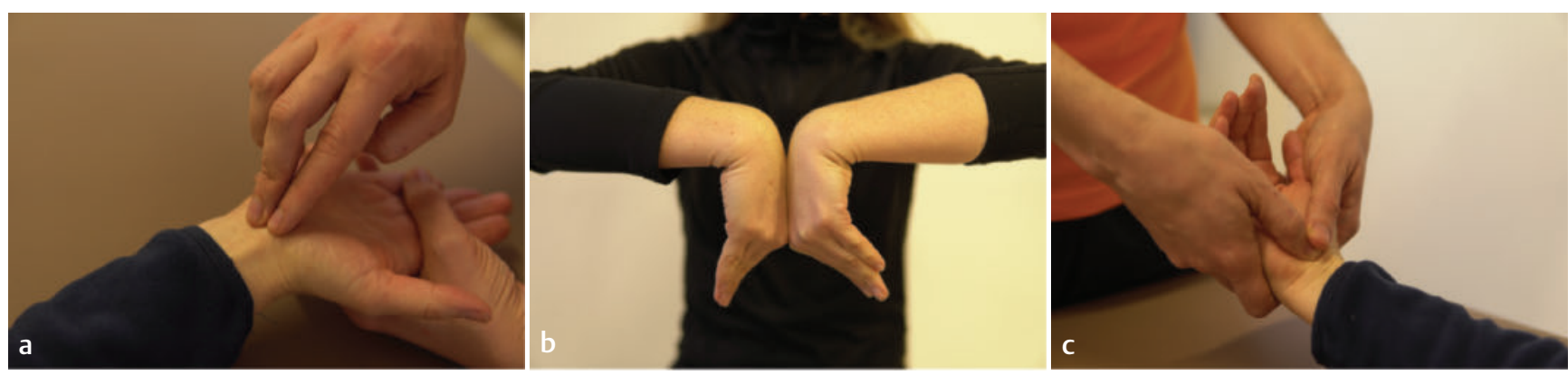

- Abb. 1 Provokationstests für den N. medianus im Karpaltunnel. a Hoffmann-Tinel-Zeichen (Beklopfen des Nerven). b Phalentest (Kompression durch Verengung des Karpaltunnelquerschnitts). c Durkan-Test (äußere Kompression durch den Untersucher). (Quelle: @ B. Neul) 
interpretieren Therapeuten dabei nicht isoliert, sondern in ihrer Gesamtheit in Form eines Testclusters [3].

Kommt es bei den beschriebenen Untersuchungsverfahren zu keinen Auffälligkeiten, führt der Therapeut neurovaskuläre Tests zur Überprüfung eines Thoracic-OutletSyndroms durch bzw. untersucht die HWS auf mögliche Bandscheibenpathologien oder Radikulopathien.

\section{Therapie}

Patienten mit Karpaltunnelsyndrom wird häufig ein chirurgischer Eingriff empfohlen, bei dem der Chirurg das Retinaculum flexorum spaltet (arthroskopisch oder offenes Verfahren). Patienten dürfen und sollen ihr Handgelenk direkt postoperativ bewegen, aber bis zu 3 Monate nicht belasten. Das Narbengewebe, welches das Retinaculum ersetzt/erweitert, muss für die anstehende Belastung erst stabil genug werden.

\section{Einem Review zufolge sollten operative Eingriffe jedoch nur dann bei Patienten vorgenommen werden, wenn diese eine Atrophie der Thenarmuskulatur aufwei- sen bzw. bereits erfolglos konservativ the- rapiert wurden [4].}

Vorrang sollten also immer Maßnahmen der Physio- und Ergotherapie bestehend aus einer Schienenversorgung, Edukation, Manueller Therapie und Bewegungsübungen haben [5]. In Bezug auf die genannten Maßnahmen fanden Fernández-de-Las Peñas et al. in ihrer Studie heraus, dass eine Operation zu keinem Zeitpunkt (1, 3, 6, 12 Monate) der Manuellen Therapie (3 Behandlungen zur Desensibilisierung und Neuromobilisation des $\mathrm{N}$.

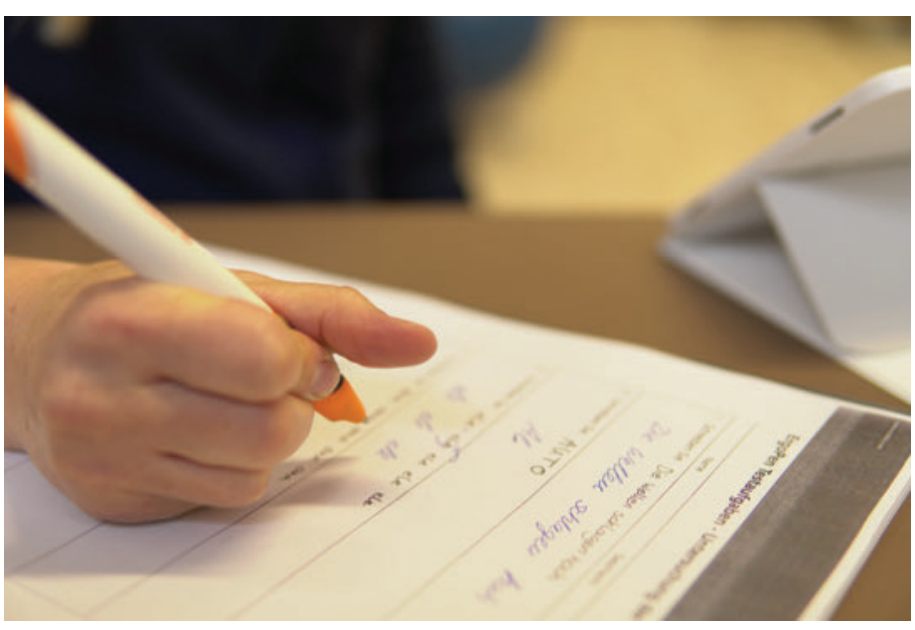

-Abb. 2 Durch die ungünstige Stifthaltung belastete Frau S. die Fingerflexoren beim Schreiben mehr als nötig. Weil sie das Daumenendgelenk in Streckung hielt, konnte der lange Daumenbeuger die Haltung des Stiftes nicht genug unterstützen. (Quelle: @ B. Neul) medianus) überlegen war [6]. Auch ein Follow-up nach 4 Jahren konnte zeigen, dass beide Verfahren gleich wirksam sind [7]. Darüber hinaus weist die Manuelle Therapie eine höhere Kosteneffektivität auf [8].

Wie ein spezifisches Therapiemanagement, bestehend aus den zuvor genannten Komponenten, aussehen kann, soll folgendes Praxisbeispiel verdeutlichen.

\section{Fallbeispiel: KTS durch \\ Schreibbelastung}

\section{Anamnese}

Frau S. ist 20 Jahre alt und studiert Psychologie. Sie stellte sich in unserer interdisziplinären Praxis für Physio- und Ergotherapie vor, da sie seit 6 Wochen vermehrt Schmerzen im rechten Handgelenk hatte. Darüber hinaus gab sie Kribbelparästhesien in den ersten 3 Fingern an, die nachts in Taubheit übergingen. Sie berichtete, dass sie in der Vergangenheit häufiger unter Sehnenscheidenentzündungen gelitten habe. Diese begannen, als sie umfangreiche Klausuren für ihr Abitur schrieb. Seither traten Schmerzen und ein Schwächegefühl nach längerem Schreiben auf. Abschließend erklärte sie, dass sie bereits therapeutische Behandlungen erhalten habe, diese ihre letzte Hoffnung sei und die Hausärztin ihr schon eine Überweisung zum Handchirurgen mitgegeben habe.

\section{Untersuchung}

Bei der Prüfung der aktiven und passiven Beweglichkeit der HWS und des Handgelenks konnten wir keine Einschränkungen feststellen oder bestehende Symptome reproduzieren. Auch eine Muskelschwäche oder Atrophie konnten wir nicht identifizieren. Ebenso unauffällig waren die Sensibilitätsprüfungen und Instabilitätstestungen im Bereich der Handwurzelknochen. Das Infrarotthermometer zeigte keine Seitendifferenz der Temperatur, sodass zu diesem Zeitpunkt kein akut entzündliches Geschehen nachzuweisen war. Durkan-Test und das Hoffmann-Tinel-Zeichen lösten die bekannten Kribbelparästhesien aus. Palpatorisch zeigte sich ein Hypertonus der Flexoren und der gesamten Thenarmuskulatur. Im DASH-Score erreichte Frau S. 35 Punkte.

Da Frau S. bereits explizit auf die Problematik der Schmerzen und des Schwächegefühls während des Schreibens hingewiesen hatte, analysierten wir die Stifthaltung und Grafomotorik genauer. Eine dauerhafte Fehlhaltung kann zur Überlastung führen und kommt somit als Ursache für die Tendovaginitis und sekundär für das KTS infrage. Bei Frau S. zeigte sich beim Schreiben schon proximal kein lockeres Handgelenk. So mussten die Finger diese fehlende Bewegung zusätzlich kompensieren. Distal zeigte sich eine steile und verkrampfte Stifthaltung mit gestrecktem Interphalangealgelenk (IP) des Daumens im Dreipunktgriff ( $\triangleright$ Abb. 2). 
Darüber hinaus führten wir einen Grafomotoriktest mit dem ErgoPen durch. Dies ist ein Stift, der mittels Biofeedback verschiedene Parameter des Schreibprozesses misst und durch Diagramme visualisieren kann. Auffällig war ein sehr geringer Automationsgrad, niedriger Schreibdruck und eine niedrige Schreibfrequenz. Es stellte sich heraus, dass sie häufiger die Haltung des Stiftes wechselte und dass sie selbst nicht mehr wusste, wie sie ihn „normalerweise“ hielt.

Auf Grundlage der ermittelten Befundparameter wählten wir die folgenden Therapiemaßnahmen aus:

\section{Schienenversorgung und Edukation}

Zunächst empfahlen wir Frau S., eine Nachtlagerungsschiene (Cook-up-Schiene) zu tragen, die das Handgelenk in einer neutralen Position hält und dadurch eine nächtliche Kompression des Nervs verhindert. Wir klärten sie auf, die Schiene nur nachts und übergangsweise zu tragen und sie mit Rückgang der Symptomatik wieder abzusetzen. Ein permanentes Tragen auch tagsüber hätte die unnatürliche Schreibbewegung fördern und somit zu einer Verstärkung der verkrampften Stifthaltung führen können. Zudem rieten wir Frau S., im Alltag darauf zu achten, ein längerfristiges Abknicken des Handgelenkes zu vermeiden, wie sie es beim Abstützen auf dem Fahrradlenker, auf der Fahrt zur Uni oder beim Lesen im Bett mit abgestützten Kopf tat.

\section{Detonisierende Maßnahmen}

Um den erhöhten Muskeltonus der Flexorengruppe zu reduzieren, setzten wir verschiedene Faszientechniken und Dehnungen ein. Diese sollen das Gewebe von Crosslinks befreien und somit eine verbesserte Gleitfähigkeit der Muskelfasern und Sehnen herstellen.

Mittels Querfriktionen arbeiten wir uns dabei vom MuskelSehnen-Übergang ( $>$ Abb. $\mathbf{3}$ ) bis zu den Sehnenscheiden vor. Ziel dabei ist einerseits die Tonussenkung der Muskulatur und andererseits, die Sehnenscheiden von allen Seiten gegenüber dem umliegenden Gewebe zu lösen, sodass diese uneingeschränkt gleiten können. Durch die Verminderung der Adhäsionen zwischen den einzelnen Blättern der Sehnenscheiden findet eine Reibungsminimierung auf die Sehne statt, was in einer geringeren Rezidivrate resultieren kann.

Unterstützend vermittelten wir Frau S. Eigenübungen: Dehnungen für die Unterarmflexoren und das Retinaculum flexorum ( $\mathbf{A} \mathbf{b b} \mathbf{4} \mathbf{4})$. Sie führte sie selbstständig zu Hause, 4x täglich je 30 Sekunden durch. Eine Studie konnte zeigen, dass die so angewandte myofasziale Eigendehnung des Retinaculum flexorum zu signifikanten Verbesserungen von Taubheit, Parästhesien und Kraft im Spitzgriff führt [9]. Für Frau S. waren die Eigendehnungen besonders leicht umzusetzen, da sie diese gut verstand und ohne großen Aufwand in ihren Alltag integrieren konnte.

\section{Nervenmobilisation}

In Kombination mit der Behandlung myofaszialer Strukturen und den entsprechenden Eigenübungen vermittelten wir Frau S. Mobilisationstechniken des N. medianus. Diese führen nachweislich zu einer Verbesserung der Symptomatik, indem intraneurale Ödeme reduziert werden [10]. Wir setzten Slider- und Tensioner-Techniken in der entsprechenden Position des Upper Limb Neurodynamic Test (ULNT) 1 bzw. 2a ein, die die Gleit- und Spannungsfähigkeit des $\mathrm{N}$. medianus verbessern können. Während beim Slider der Nerv an einem Ende (Dorsalextension der Hand) auf Spannung und am anderen Ende (ipsilaterale Seitneigung der HWS) entspannt wird, kommt es beim Tensioner zu einer Spannungserhöhung auf beiden Seiten (kontralaterale Seitneigung der HWS).

Die neuralen Mobilisationstechniken bereiteten Frau S. aufgrund der wechselnden Kopf- und Armbewegungen zunächst koordinative Probleme, sodass wir die Tensioner-Ausführung ( $\mathbf{A b b}$. 5) als Eigenübung festlegten.

\section{Anbahnen neuer Bewegungsmuster und Kräftigung}

Neben der Kräftigung der Handgelenksextensoren sollte die Patientin lernen, den M. flexor pollicis longus (FPL) gezielt einzusetzen, da sie ihn in der bestehenden Stifthaltung nicht nutzte. Der FPL verläuft zwar durch den Karpaltunnel, jedoch im 2. palmaren Sehnenfach, während FDS und FDP das 3. Sehnenfach durchqueren.

Ziel war es also, die hypertonen Fingerflexoren beim Schreiben zu entlasten und dadurch zur Korrektur der Grafomotorik beizutragen. Um den FPL gezielt zu trainieren, nutzten wir das Brunelli-Plättchen insbesondere beim Einsatz von feinmotorischen Spielen (> Abb. 6$)$. Zu

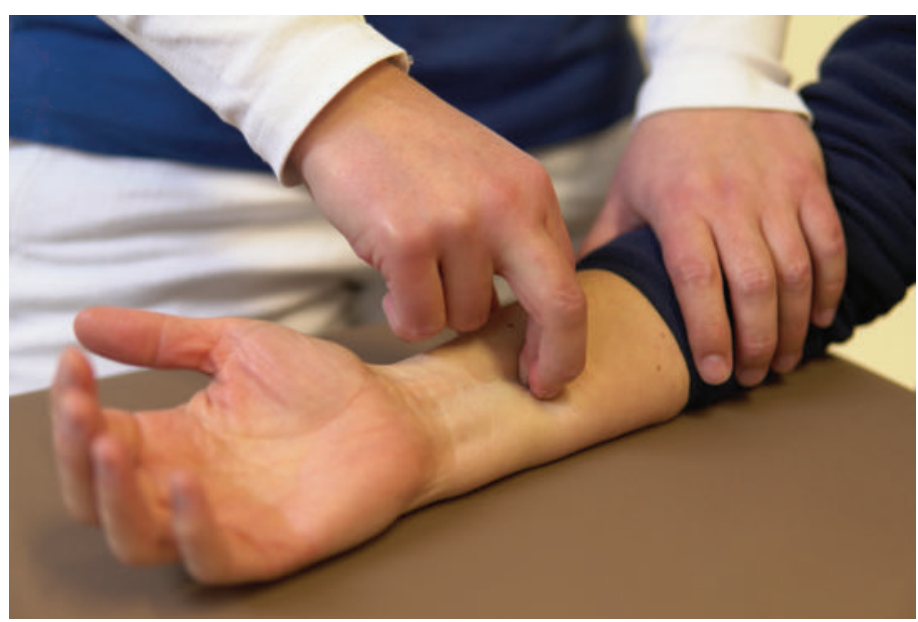

-Abb. 3 Therapeuten können Querfriktion bei Sehnenscheidenentzündungen zur Tonussenkung und Adhäsionslösung einsetzen. (Quelle: @ B. Neul) 


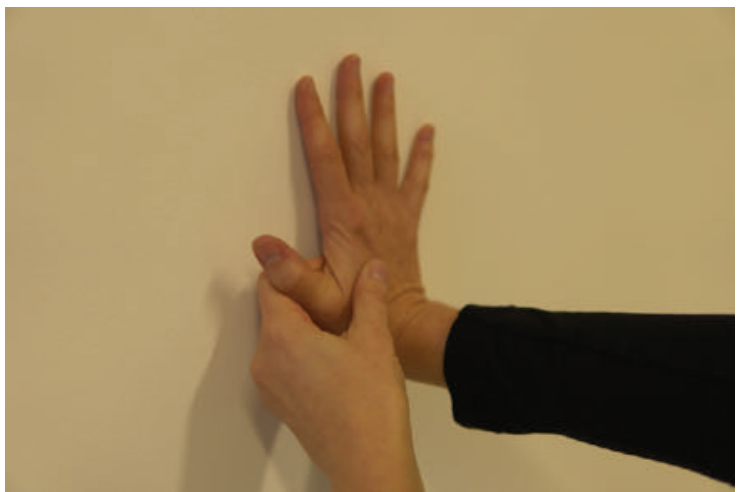

-Abb. 4 Die myofasziale Dehnung des Retinaculum flexorum eignet sich optimal als Eigenübung. (Quelle: @ B. Neul)

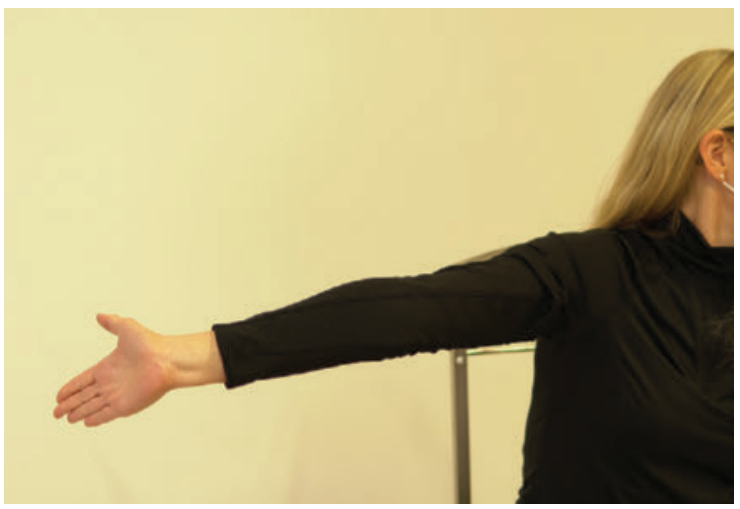

- Abb. 5 Der Tensioner in Eigenübung. Durch die Haltung des Armes wird der N. medianus auf Spannung gebracht. (Quelle: @ B. Neul)

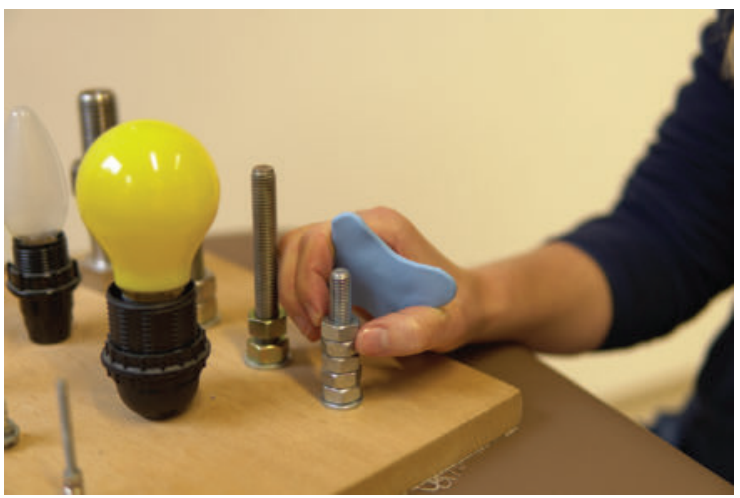

- Abb. 6 Bei der Übung mit dem Brunelli-Plättchen flektiert die Patientin gezielt das Daumenendgelenk und steuert so den $\mathrm{M}$. flexor pollicis longus verstärkt an. (Quelle: @ B. Neul)
Beginn ohne, später mit Widerstand (z. B. Klammern). Im Anschluss an die Therapie und auch zu Hause können zur Automatisierung der Bewegungsabläufe Übungen ohne Brunelli-Plättchen und Widerstand im warmen Rapsbad erfolgen.

\section{Ergonomieberatung und Grafomotorikschulung}

Wir testeten verschiedene Stiftadaptionen, um eine physiologische Schreibposition zu erzielen. Frau S. erhielt daraufhin eine Schreibhilfe die ihre steile Stifthaltung verbesserte und die es ihr ermöglichte, das IP-Gelenk flektiert zu halten und weniger Druck auf den Stift zu bringen. Zu viel Druck ist an einem weißen Daumennagel zu erkennen. Die Patientin bekam den Auftrag, die Stiftadaption im Alltag und bei längeren Schreibprozessen einzusetzen. Dies fiel ihr insbesondere zu Beginn aufgrund der Umstellung des Schreibverhaltens schwer. Wir zeigten der Patientin in dieser Phase ihre bereits erzielten Verbesserungen auf, um sie zur weiteren Umgewöhnung des Schreibens zu motivieren.

Für eine dauerhafte Veränderung kann es sinnvoll sein, zusätzlich ein Grafomotoriktraining zu durchlaufen. Hier wird z. B. mittels Schwungübungen für das Handgelenk der Schreibprozess neu aufgesetzt und beübt.

\section{Zwischenbefund}

Nach 6 Behandlungen hatte sich der DASH-Index um 15 Punkte verbessert. Laut der Patientin treten die nächtlichen Missempfindungen zu diesem Zeitpunkt seltener und in reduzierter Intensität auf. Des Weiteren gab sie an, mit der Stiftadadaption längere Zeit ohne Schmerzen schreiben zu können.

\section{Fazit}

Zur Behandlung des Karpaltunnelsyndroms sollten zunächst konservative Behandlungsmaßnahmen in Betracht gezogen werden. Neben der Schienenversorgung, manuellen Therapieverfahren und Eigenübungen ist es empfehlenswert, auch Beratungen zu Ergonomie und Grafomotorik in ein interdisziplinäres Therapiemanagement zu integrieren. Physiotherapeuten und Ergotherapeuten können hierbei gemeinsam Hand in Hand agieren, um Patienten eine effektive Therapie zu ermöglichen. 


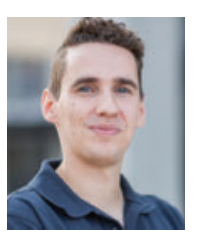

\section{Benjamin Neul}

Physiotherapeut und leitender Angestellter in der Praxis für Ergo- und Physiotherapie Jensen in Gießen. Er unterrichtet zudem als Dozent an der Ludwig-Fresenius-Schule Marburg und hat einen Lehrauftrag an der DIPLOMA-Hochschule. Darüber hinaus ist er Gründer und Geschäftsführer der MoveYourMind GmbH.

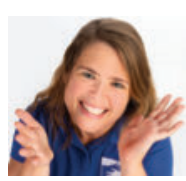

\section{Carina Jensen}

Ergotherapeutin und Praxisinhaberin zweier Praxen für Handtherapie, Ergotherapie und Physiotherapie in Gießen und Lich-Eberstadt (Hessen). Sie ist unter anderem zertifizierte Handtherapeutin der AFH, Manualtherapeutin für die obere Extremität und Referentin bei Handkursen und auf Handsymposien.

\section{Korrespondenzadresse}

Ergo- und Physiotherapie Jensen

Schiffenberger Weg 61

35394 Gießen

Deutschland

E-Mail: info@ergo-handtherapie.com

www.ergo-handtherapie.com

\section{Literatur}

[1] Rosenbaum RB, Ochoa JL, Hrsg. Carpal tunnel syndrome and other disorders of the median nerve. Woburn: ButterworthHeinemann; 2002

[2] Erickson M, Lawrence M, Jansen CWS et al. Hand pain and sensory deficits: Carpal tunnel syndrome. J Orthop Sports Phys Ther 2019; 49: CPG1-CPG85

[3] JOSPT. Carpal tunnel syndrome: A summary of clinical practice guideline recommendations-Using the evidence to guide physical therapist practice. J Orthop Sports Phys Ther 2019; 49(5): $359-360$

[4] Klokkari D, Mamais I. Effectiveness of surgical versus conservative treatment for carpal tunnel syndrome: A systematic review, meta-analysis and qualitative analysis. Hong Kong Physiother J 2018; 38(2): 91-114

[5] Lewis KJ, Coppieters MW, Ross L et al. Group education, night splinting and home exercises reduce conversion to surgery for carpal tunnel syndrome: A multicentre randomised trial. J Physiother 2020; 66(2): 97-104

[6] Fernández-de-Las-Peñas C, Ortega-Santiago R, de la Llave-Rincón Al et al. Manual physical therapy versus surgery for carpal tunnel syndrome: A randomized parallel-group trial. J Pain 2015; 16: 1087-1094
[7] Fernández-de-Las-Peñas C, Aria-Buría JL, Cleland JA et al. Manual therapy versus surgery for carpal tunnel syndrome: 4year follow-up from a randomized controlled trial. Phys Ther 2020; 100(11): 1987-1996

[8] Fernández-de-Las-Peñas C, Ortega-Santiago R, Saddi Díaz HF et al. Cost-effectiveness evaluation of manual physical therapy versus surgery for carpal tunnel syndrome: Evidence from a randomized clinical trial. J Orthop Sports Phys 2019; 49(2): 55-63

[9] Shem K, Wong J, Dirlikov B. Effective self-stretching of carpal ligament for the treatment of carpal tunnel syndrome: A double-blinded randomized controlled study. J Hand Ther 2020; 33: $272-280$

[10] Paquette P, Higgins J, Gagnon DH. Peripheral and central adaptations after a median nerve neuromobilization program completed by individuals with carpal tunnel syndrome: An exploratory mechanistic study using musculoskeletal ultrasound imaging and transcranial magnetic stimulation. J Manipulative Physiol Ther 2020; 43(6): 566-578 